Portland State University

PDXScholar

\title{
Modeling and Visualizing Power Amplification in Fiber Optic Cables
}

\section{Gil Parnon}

Portland State University

Follow this and additional works at: https://pdxscholar.library.pdx.edu/honorstheses

Part of the Mathematics Commons

\section{Let us know how access to this document benefits you.}

\section{Recommended Citation}

Parnon, Gil, "Modeling and Visualizing Power Amplification in Fiber Optic Cables" (2020). University Honors Theses. Paper 830.

https://doi.org/10.15760/honors.849

This Thesis is brought to you for free and open access. It has been accepted for inclusion in University Honors Theses by an authorized administrator of PDXScholar. Please contact us if we can make this document more accessible: pdxscholar@pdx.edu. 
Modeling and Visualizing Power Amplification In Fiber Optic Cables

by

Gil Parnon

An undergraduate honors thesis submitted in partial fulfillment of the requirements for the degree of

Bachelor of Science

in

University Honors

and

Mathematics

Thesis Adviser

Dr. Jay Gopalakrishnan

Portland State University

2020 


\begin{abstract}
Transverse mode instability in fiber optic cables causes power amplification to exhibit chaotic behavior. Numerical modeling of fiber optic power amplification is extremely computationally expensive. In this paper I work through modeling similar behavior in a simpler system. I also visualize the three-dimensional phase portrait of the system in order to better understand the behavior and hopefully relate it to more well-understood problems.
\end{abstract}

\title{
1 Background
}

optical fiber is essentially a glass tube roughly the thickness of a human hair that can be used to transmit light. Due to the properties of the glass used (and the chemical processes that modify the glass) the index of refraction keeps light trapped inside as it is transmitted. This allows for a light to be shined into one end of the cable and have that transmit to the other end without it 'coming out the sides of the cable'. Also, despite being glass the cables are flexible and fairly sturdy. optical fiber cables are commonly used for various networking applications such as running internet lines as well as phone lines [7].

Despite being more expensive, optical fiber has begun to replace copper wire for a lot of applications for a number of reasons. The first is that it is physically lighter as well as being more resilient. The second major reason is that optical fiber can transmit over significantly longer distances with no signal degradation. The cables also do not have to worry about interference from electromagnetic fields. They also do not generate as much heat as a copper wire with current running through it, and are harder to hack into without being detected [4]. There are still some issues with power scaling of optical fiber amplifiers however, as the signal is amplified significantly it begins to develop chaotic behavior, this is called transverse mode instability (TMI) [3].

From [3] the simulation time can be cut by roughly a factor of 100 without increasing error too dramatically. The problem is that these simulations still take quite a while to run. The goal of this project is to relate the nonlinear power exchanges, observed from experiments and long simulations, to simpler well-known nonlinearities like the predator-prey system. This helps us develop a better intuition for the causes of power exchange.

\section{Methods}

This system will need to model the coupled behavior between the index of refraction and the temperature of the section of optical fiber cable. As temperature increases the index of refraction changes which then changes how the light reflects inside the cable. This in turn changes the temperature so that you have a coupled system. We can develop quite complex models to understand this system, unfortunately they are far too computationally expensive to actually 
simulate. A slightly simpler system can be found in [3], but it still requires a significant amount of time to generate a solution for a short piece of cable.

The model we will be building is based on a model from [3]. This is derived from coupled mode theory or CMT. The CMT model computes the amplitude of the pump power, as well as several guided modes. These are called linearly polarized transverse guided core modes [1]. For the rest of this paper, I will refer to them as LP modes. Each mode is a function $\varphi(x, y)$ which decays exponentially at the boundary and satisfies

$$
\left(\Delta_{x y}+k_{\ell}^{2} n^{2}\right) \varphi_{m}=\beta_{m}^{2} \varphi_{m}, m=1, \ldots, M_{\ell}
$$

for $\beta_{m}$ is the appropriate propagation constant, and $\Delta_{x y}=\partial_{x x}+\partial_{y y}$, the transverse Laplacian operator [3]. The electric field can be expressed as a scalar Helmholtz equation, which can be expressed in terms of $\varphi$ and its corresponding complex field amplitudes, $A_{m}(z)[3]$.

$$
\overrightarrow{E_{\ell}}=U_{\ell} \hat{e_{x}}=\sum_{m=1}^{M_{\ell}} A_{m}^{\ell}(z) \varphi_{m}(x, y) e^{i \beta_{m} z}
$$

where $\hat{e_{x}}$ is the unit vector in the $x$-direction. From [3] the full CMT model computes

$$
Y(z)=\left[I_{p}(z), A_{1}(z), A_{2}(z), \ldots, A_{M}(z)\right]^{t}, \text { for } 0<z<L,
$$

where I is the irradiance of the pump, and $A_{m}(z)$ is the $m^{\text {th }}$ signal mode amplitude. Y satisfies the following system of ODEs:

$$
\frac{d Y}{d z}=\left[\begin{array}{cc}
\left\langle g_{p}(Y)\right\rangle & 0 \\
0 & \phi(z) \cdot K(Y)
\end{array}\right] Y
$$

where $\phi$ is a matrix defined by $\phi_{l m}(z)=e^{i\left(\beta_{m}-\beta_{l}\right) z}$ and $\phi(z) \cdot K(Y)$ is the Hadamard product of $\phi$ and $K$. K is a mode coupling coefficient which requires an integral at each step. $g_{p}$ is an active gain term. $\left\langle g_{p}(Y)\right\rangle$ is the mean value of $g_{p}$ over a given cross section of the optical fiber.

For now, most of my work will be based on the following differential equation where $\mathrm{P}$ is the power for the fiber [3]. This comes from a further treatment of the CMT model above. In this system of equations, $\mathrm{P}$ is a vector valued function where $P_{i}$ is the $i^{t h}$ mode of the power, and $P_{1}$ is the power from the pump.:

$$
\frac{d P}{d z}=\left[\begin{array}{cc}
\left\langle g_{p}(Y)\right\rangle & 0 \\
0 & 2 \operatorname{diag}[K(Y)]
\end{array}\right] P+\left[\begin{array}{c}
0 \\
\rho(Y)
\end{array}\right]
$$

In my case, we will be restricting this to a $3 \times 3$ matrix. We will have the pump power and then two modes from the signal. In Figure 1 below you can see the ideal situation which leads to a properly boosted signal.

In Figure 2 you can see the issue where the $2^{\text {nd }}$ mode of the signal begins to take some of the power leading to an unstable and inconsistent power level in the signal. The source of this instability is not well understood, and my 

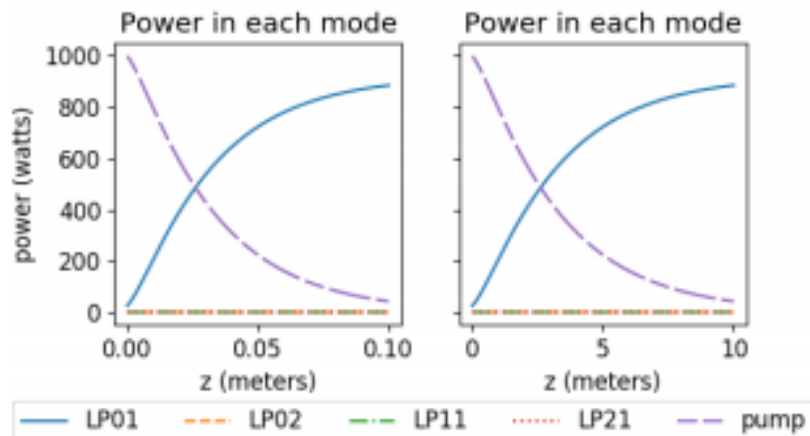

Figure 1: Figure reproduced from [3] showing the exchange of power between the pump and signal
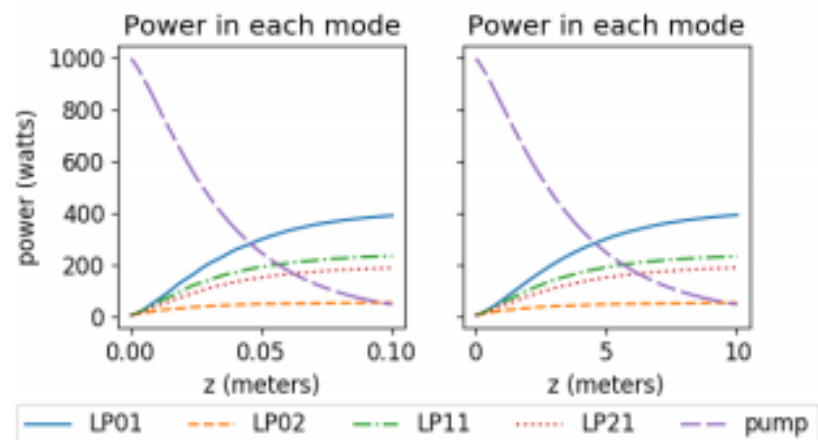

Figure 2: Figure reproduced from [3] showing how the secondary modes begin to take power from the fundamental mode

paper will be focusing on developing a . In equation (1) the matrix component is a nonlinearity. For the sake of simplicity, we will be ignoring the $\left[\begin{array}{c}0 \\ \rho(Y)\end{array}\right]$ term. This will allow me to exclusively investigate the non-linear aspect of this problem. By testing out a variety of different nonlinearities, taken from physical problems [6] we will hopefully be able to gain some intuition into the effects that these different nonlinear terms have on the system. This in turn can hopefully shed light on the more complicated model in order to better understand what causes TMI.

My model will be a small system along the lines of

$$
\frac{d P}{d z}=\left[\begin{array}{ccc}
K_{11}\left(P_{0}, P_{1}, P_{2}\right) & K_{12}\left(P_{0}, P_{1}, P_{2}\right) & K_{13}\left(P_{0}, P_{1}, P_{2}\right) \\
\cdot & \cdot & \cdot \\
\cdot & \cdot & K_{33}\left(P_{0}, P_{1}, P_{2}\right)
\end{array}\right] P
$$


where $P$ is the vector $\left[\begin{array}{l}P_{\text {pump }} \\ P_{L P 01} \\ P_{L P 11}\end{array}\right]$ with initial conditions $\left[\begin{array}{c}1000 \\ 25 \\ 0\end{array}\right]$.

\section{Results}

For accomplishing the previously stated goal we used commonly found nonlinearities to define $K_{i j}$ and catalog the resulting $P_{i}$. From the figure above it is clear that the pump power decreases at a rate proportional to the increase in the signal. The term $P_{L P 01}$ seems to increase as a function of the pump power, while decreasing as a function of $P_{L P 11}$. Finally we had $P_{L P 11}$ increase proportionally to $P_{L P 01}$ as shown below.

$$
\frac{d P}{d z}=\left[\begin{array}{ccc}
-P_{L P 01}-P_{L P 11} & 0 & 0 \\
0 & P_{\text {pump }}-P_{L P 11} & 0 \\
0 & 0 & P_{L P 01}
\end{array}\right] P .
$$

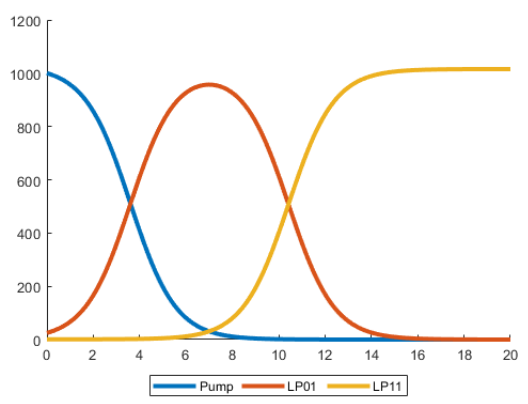

Figure 3: System 7 numerically simulated using RK4 on $[0,20]$ with a step size of 0.1 
Somewhat predictably, the steady state ends up with $P_{L P 11}$ being the amplified power as it does not have a term which causes it to decrease so it will rise until the pump power and $P_{L P 01}$ are 0 . However you can see that in the real case the power in each mode is a monotonically increasing function, so $P_{L P 11}$ should not detract from $P_{L P 01}$, merely reduce the rate at which $P_{L P 01}$ increases as there is not as much power to take for itself. Therefore we consider

$$
\frac{d P}{d z}=\left[\begin{array}{ccc}
-P_{L P 01}-P_{L P 11} & 0 & 0 \\
0 & P_{\text {pump }} & 0 \\
0 & 0 & P_{\text {pump }}
\end{array}\right] P .
$$

Equation 8 reflects this change. There should be no oscillation at all, they will monotonically increase. This simulation seemed more reasonable as you can see in Figure 4. This much more closely resembles the system in Figure 2 , and with some weighting you could see how this will very closely resemble that situation. By weighting $P_{L P 01}$ by a factor of 0.6 in the system we get (9). This clearly still needs some work, as the decrease of pump power needs to be exponential however it briefly lags due to the initial conditions having small values for $P_{L P 01}$ and $P_{L P 11}$.

$$
\frac{d P}{d z}=\left[\begin{array}{ccc}
-P_{L P 01}-P_{L P 11} & 0 & 0 \\
0 & 0.6 * P_{\text {pump }} & 0 \\
0 & 0 & P_{\text {pump }}
\end{array}\right] P .
$$

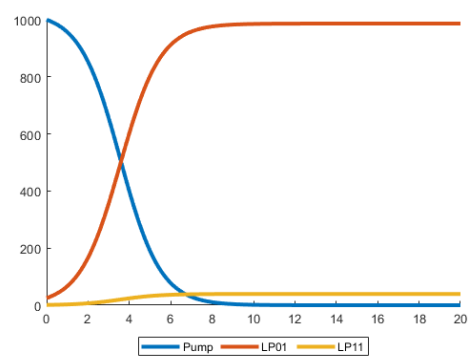

(a) System (8)

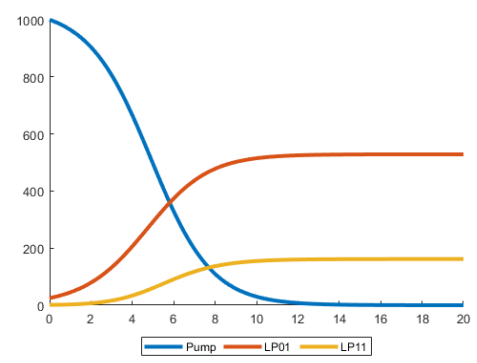

(b) The weighted system (9)

Figure 4: Redone systems using the same simulation parameters as before 
From here we were trying to find other possible small systems with chaotic behavior. We looked into the Lorenz Attractor, a fairly simple deterministic system known for its chaotic behavior. Behaviors oscillate around two attractor points. This system comes from Edward Lorenz in 1963 [5]. He was trying to model the weather. When he tried to restart a simulation from a halfway point using given values at that point he found that it diverged to a different solution. This is because the computer was using six digits to simulate, but only outputted three, and the error was enough to cause significant difference in the solutions. This idea was the birth of modern chaos theory. Lorenz described it by saying, "Chaos: When the present determines the future, but the approximate present does not approximately determine the future" [2]. From this, Lorenz was able to determine that we will most likely never be able to approximate the weather past two weeks due to the inherently nonlinear nature of nature. The phase portrait of this system aesthetically resembles a butterfly, giving rise to the concept of the butterfly effect, that small changes to initial conditions will lead to drastic change. From viewing the Lorenz attractor, we wanted to determine if there was a possibility that the fiber optic cable power levels could exhibit some properties of a strange attractor.

$$
\left\{\begin{array}{l}
\frac{d x}{d t}=\sigma(y-x) \\
\frac{d y}{d t}=x(\rho-z)-y \\
\frac{d z}{d t}=x y-\beta z
\end{array}\right.
$$

We took the data from the simulations for two milliseconds. From this we generated a video of the animated phase portrait for both the Thulium doped fiber, as well as the Ytterbium doped fiber. The goal was to be able to see if there was any kind of strange attractor properties that it exhibited or converged to. The snapshot below of the Thulium doped cable is fairly typical for what we saw for the Thulium.

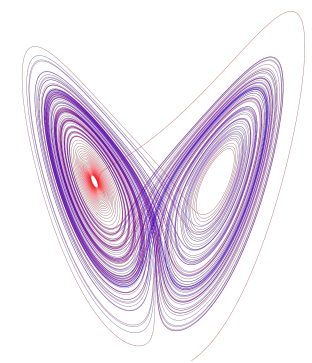

(a) The Lorenz Attractor

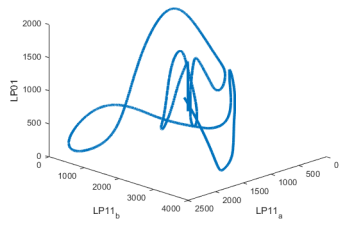

(b) Thulium Doped Fiber Optic Cable

Figure 5: Redone systems using the same simulation parameters as before

The curve is seemingly random, and flails around with no discernible pattern. The same is true with the Ytterbium doped cable, albeit with more noise. We conclude that any chaotic behaviour in the power system for fiber amplifiers is not likely to yield a strange attractor set like that of Lorenz. 


\section{References}

[1] Govind Agrawal. Nonlinear Fiber Optics. Academic Press, 2013.

[2] Chaos in an Atmosphere Hanging on a Wall. URL: http://mpe.dimacs. rutgers . edu/2013/03/17/chaos-in-an-atmosphere-hanging-on-awall/. (accessed: 3.18.2020).

[3] D. Drake et al. "Simulation of Optical Fiber Amplifier Gain Using Equivalent Short Fibers". In: Computer Methods in Applied Mechanics and Engineering 7.11 (2013), pp. 861-867.

[4] Fibre (Fiber) vs Copper as Fast as Possible. URL: https://www. youtube. com/watch?v=_Bw2NFBDxR8. (accessed: 11.18.2019).

[5] Edward Norton Lorenz. "Deterministic nonperiodic flow". In: Journal of the Atmospheric Sciences 20 (1963), pp. 130-141.

[6] Steven H Strogatz. Nonlinear Dynamics and Chaos: with Applications to Physics, Biology, Chemistry, and Engineering. Westview Press, 2015.

[7] The FOA Reference for Fiber Optics - Optical Fiber. URL: http://www . thefoa.org/tech/ref/basic/fiber.html. (accessed: 11.18.2019). 